\section{Isolation effect based on digit composition of numbers and its stability}

\author{
STEFAN SLAK, University of Toledo, \\ Toledo, Ohio 43606
}

Forty Ss were given a serial-learning task involving 10 three-place numbers. The sixth item either had similar digit composition or was isolated by having a different digit composition. A strong and stable isolation effect was observed. The isolation effect was interpreted as being due to a lesser degree of formal similarity, and its stability to the absence of spread of isolation effect.

Wallace (1965) reviewed types and characteristics of isolation effect, indicating two major ways of isolating an item: (1) by an additional operation (e.g, different color), and (2) by selecting a different type of item (e.g., a nonsense syllable embedded in a list of words), with either the isolated and the control item or the background material being the same in the two conditions. Many studies, however, indicate that formal similarity of the same type of items consistently results in a decreased performance in various serial- and free-learning and recall tasks (e.g., Underwood, 1952; Stimmel \& Stimmel, 1967; Slak, 1969). It might be expected that a decrease in formal similarity of an item with other items will result in an isolation effect, though the type of item is the same. The purpose of the present study is to test this hypothesis.

\section{METHOD}

Forty undergraduate students were assigned randomly to experimental conditions.

Four different serial lists of 10 three-place numbers were used. They were identical except for the sixth item. The order of items was: $143,231,423,312,124$, the sixth, $234,314,431,142$. These items were composed of digits 1 to 4 . Each list had a different sixth item. In two control lists, these items were composed of the same selection of digits (341 and 243), whereas the two items in the sixth position in the experimental lists were composed of a different selection of digits (896 and 798).
Each of the four lists was used in a group of $10 \mathrm{Ss}$ and was presented at a 2 -sec rate for a total of 15 trials, each trial being followed by a 30 -sec serial-reproduction period. RESULTS

For each $S$, the total number of correct responses was determined for each serial position in all trials. An item in a given serial position was considered correctly recalled if it appeared on the answer sheet in any position.

The results for the two control lists and those for the two experimental lists were pooled because of the absence of any significant difference, either in terms of the entire curve or in the sixth position. The serial-position curves for the pooled control and pooled experimental groups are presented in Fig. 1.

\section{Isolation Effect}

The recall score on the isolated item was significantly higher than it was for the control item in the same position $(t=2.519$, $\mathrm{df}=38, \mathrm{p}<.01$ ). Though the effect is quite strong, inspection of Fig. 1 does not suggest any spread of isolation effect to adjacent items. It is also apparent that isolation effect was stronger than primacy effect in the isolated condition and as strong as primacy effect in the control condition.

$$
\text { Recall of Massed Items }
$$

A 2 by 9 analysis, with factors of condition and serial position and repeated measures on the last factor, was performed to evaluate the overall difference between the two curves after the subtraction of the sixth item (yielding nine remaining serial positions). Serial-position effect was significant $(F=9.633, \mathrm{df}=8 / 304, \mathrm{p}<.01)$, but the effect of conditions $(F=2.416$, $\mathrm{df}=1 / 38, p>.05)$ and the interaction between Conditions and Serial Position $(F=0.455$, df $=8 / 304, p>.05)$ were not significant.

Stability of Isolation Effect

Variances for the six th position were 4.00 for the isolated item and 15.94 for the control item. The difference was significant $(F=3.985, \quad \mathrm{df}=19 / 19, \quad \mathrm{p}<.01)$. This shows a remarkable stability of the isolation

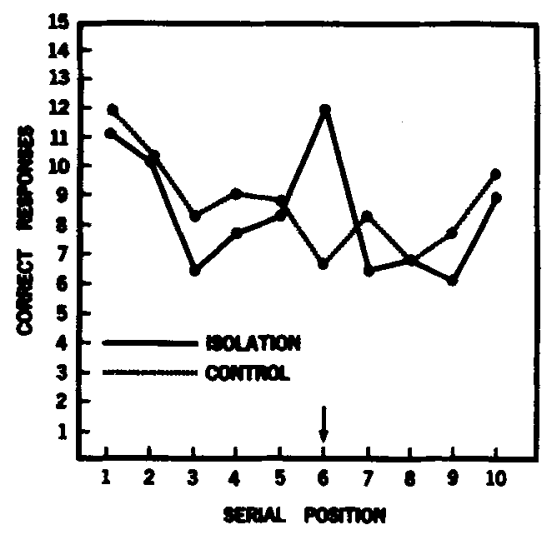

Fig. 1. Serial-position curves for the isolation and control conditions.

effect that can, in part, be explained as the result of a ceiling effect. However, the score on the first item in the control group is equally close to the ceiling as the score on the isolated item, but its variance of 8.947 is again greater than the variance of the isolated item $(F=2.237, d f=19 / 19$, $p<.05)$. Stability of the isolation effect is not entirely due to the ceiling effect.

\section{DISCUSSION}

The results show that a decrease in formal similarity of an item with other items, based on a different digit composition, results in a rather strong isolation effect. The isolated item does not have to belong to a different category.

More unexpected was the remarkable stability of the isolation effect, going beyond what might be expected from the ceiling effect. Such stability is accompanied by a complete absence of any spread of effect. It could be argued that the spread of isolation effect would result in a certain distribution of attention over several items and fluctuation of attention with respect to the isolated item, with an increase in variance of recall score. The stability of the isolation effect may result from the absence of such fluctuation. This explanation is supported by a comparison with the primacy effect, where the spread of effect is a rule.

\section{REFERENCES}

SLAK, S. Information, similarity and vocalization in serial learning of digits. Perceptual \& Motor Skills, 1969, 28, 311-316.

STIMMEL, D. T., \& STIMMEL, N.S. Free recall as a function of intralist similarity and order of presentation constraints. Psychological Reports, 1967, 21, 541-544.

UNDERWOOD, B. J. Studies of distributed practice: VII. Learning and retention of serial nonsense lists as a function of intralist similarity. Joumal of Experimental Psychology, $1952,44,80-87$.

WALLACE, W. P. Review of the historical, empirical, and theoretical status of the von Restorff phenonenon. Psychological Bulletin, 1965, 63, 410-424. 